\title{
Editorial
}

\section{Managing cardiovascular risk in type 2 diabetes}

\author{
Sri Lanka Journal of Diabetes, Endocrinology and Metabolism 2012; 2: 1-2
}

Diabetes is associated with a marked increase (by a factor of two to four) in the risk of coronary heart disease (1-3). Clinically established coronary heart disease itself is associated with an increase in mortality from coronary heart disease by a factor of three to seven, depending on the mode of presentation $(4,5)$. Among patients with type 2 diabetes, the seven year risk of suffering a first myocardial infarction (MI) is similar to that of recurrent MI in non diabetic persons who have had a previous MI (6).

The presence of coronary artery disease is 10 times greater among patients with type 1 diabetes than age and gender matched persons without diabetes (7). Cardiometabolic risk factors, including insulin resistance and associated manifestations, predispose to increased cardiovascular disease (CVD) in type 2 diabetes.

Glycaemic control with HbA1c targets of less than 7 resulted in 50\% reduction in the incidence of CVD in patients with type 1 diabetes enrolled in the DCCT/EDIC study (8). The UKPDS post-trial monitoring results showed continuing benefit of earlier intensive glycaemic control with maintenance of the relative risk reductions for any diabetes related endpoint (21\%, $\mathrm{P}=0.013)$, myocardial infarction (33\%, $\mathrm{P}=0.005)$ and all-cause mortality (27\%, $\mathrm{P}=0.002)$, despite loss of within trial blood glucose and antihyperglycaemic therapy differences - a legacy effect of type 2 diabetes (9).

In contrast, several recent studies (ACCORD (10), VADT (11), ADVANCE (12) have failed to demonstrate CVD risk reduction through intensive glycaemic control in patients with type 2 diabetes. Therefore, the comprehensive approach that recognizes and controls multiple CVD risk factors is the most effective management strategy in patients with diabetes (13).

In all patients with diabetes, cardiovascular risk factors should be assessed at least annually. The risk factors include dyslipidaemia, hypertension, smoking, positive family history of premature coronary disease and the presence of micro or macroalbuminuria. Abnormal risk factors should be treated to recommended targets (14).

Effective life style intervention improves glycaemic control, blood pressure, lipids and associated cardio metabolic risk factors. Preliminary data of the Look AHEAD Study indicate benefits in CVD risk markers associated with weight loss (15). Smoking cessation should be promoted for all patients who are smoking in order to reduce the CVD risk. Patients with diabetes should achieve target lipid levels. In the lipid arm of the ACCORD (10) trial, addition of fenofibrate to raise HDL and decrease triglycerides in patients taking simvastatin did not reduce the rate of fatal cardiovascular events, non fatal MI or non fatal stroke as compared with simvastatin alone. These results do not support the routine use of combination therapy with fenofibrate and simvastation to reduce the CVD risk in the majority of high risk patients with type 2 diabetes.

In the patients with type 2 diabetes at high risk for CVD, targeting a systolic blood pressure of less than 120 $\mathrm{mmHg}$, as compared with less than $140 \mathrm{mmHg}$, did not reduce the rate of a composite primary outcome of fatal and non fatal major CVD events [ACCORD study (10)]. Therefore, patients with diabetes blood pressure target of 130/80 mmHg should be achieved to reduce the CVD risk in diabetes until further evidence is available.

Recent randomised controlled trials have failed to show CVD benefits of aspirin use for primary prevention in patients with diabetes (15). Low dose aspirin for primary prevention is reasonable for adults with diabetes and no previous history of vascular disease who are at increased CVD risk (10 year risk of CVD events more than 10\%) and who have no known risk for bleeding. Appropriate candidates include most men over 50 years of age and women aged over 60 years with major risk factors (15). Aspirin is recommended in secondary prevention of diabetes patients with documented CVD events. In patients with known CVD, ACE inhibitor, aspirin and statin therapy should be used to reduce the cardiovascular events.

In conclusion, a comprehensive approach to the prevention and management of heart disease in diabetes patients is advocated. This is best accomplished through a combination of life style modification and targeting of the multiple cardio metabolic risk factors and co morbidities.

- Dr. Uditha Bulugahapitiya

\section{References}

1. Kannel WB, McGee DL. Diabetes and glucose tolerance as risk factors for cardiovascular disease: the Framingham Study. Diabetes Care 1979; 2: 120 - 6.

2. Pyorala K, Laakso M, Uusitupa M. Diabetes and atherosclerosis: an epidemiologic view. Diabetes Metab Rev 1987; 3: 463 - 524.

3. Stamler J, Vaccaro O, Neaton JD, Wentworth D. Diabetes, other risk factors, and 12-yr cardiovascular mortality for men screened in the Multiple Risk Factor Intervention Trial. Diabetes Care 1993; 16: 434 - 44. 
4. Pekkanen J, Linn S, Heiss G, et al. Ten-year mortality from cardiovascular disease in relation to cholesterol level among men with and without preexisting cardiovascular disease. $N$ Engl J Med.

5. Rosengren A, Hagman M, Wedel H, Wilhelmsen L. Serum cholesterol and long term prognosis in middle-aged men with myocardial infarction and angina pectoris: a 16-year follow up of the Primary Prevention Study in Göteborg, Sweden. Eur Heart J 1997; 18: 754 - 61.

6. Haffner SM, Lehto S, Ronnemaa T, et al. Mortality from coronary heart disease in subjects with type 2 diabetes and in non diabetic subjects with or without prior myocardial infarction. N Engl J Med 1998; 339: 229-34.

7. Laing SP, Swerdlow AJ, Slater SD, et al. Mortality from heart disease in a cohort of 23000 patients with insulin treated diabetes. Diabetologia 2003; 46: 760 - 5.

8. DCCT/EDIC Research Group. Intensive diabetes treatment and cardiovascular disease in type 1 diabetes in the DCCT/ EDIC. N Engl J Med 2005; 353: 2643 - 53.

9. Holman RR, Paul SK, Bethel MA, Matthews DR, Neil HAW. 10-year follow up of intensive glucose control in type 2 diabetes. $N$ Engl J Med 2008; 359: 1577- 89.
10. The ACCORD Study Group. Effects of intensive blood pressure control in type 2 diabetes. $N$ Engl J Med 2010; 362: 1575 - 85 .

11. Duckworth W, Abraira C, Moritz T, et al. VADT Investigators. Glucose control and vascular complications in veterans with type 2 diabetes. $N$ Engl J Med 2009; 360: 129 - 39.

12. The ADVANCE Collaborative Group. Intensive blood glucose control and vascular outcomes in patients with type 2 diabetes. $N$ Engl J Med 2008; 358: 2560 - 72.

13. Gaede P, Lund-Anderson H, Parving HH, Pedersen O. Effect of a multifactorial intervention on mortality in type 2 diabetes. N Engl J Med 2008; 358: 580 - 91.

14. Redmon JB, Bertonia G, Connelly S, Feeney PA, et al. Effect of the Look Ahead Study intervention on medication use and related cost to treat cardiovascular disease risk factors in individuals with type 2 diabetes. Diabetes Care 2010, 33: 1153 - 8.

15. Pignone M, Alberts MJ, Colwell JA, et al. Aspirin for primary prevention of cardiovascular events in people with diabetes. Diabetes Care 2010; 33: 1395 - 402. 